\title{
FREQUENCY ANALYSIS USING NON-UNIFORM SAMPLING WITH APPLICATION TO ACTIVE QUEUE MANAGEMENT
}

\author{
Frida Gunnarsson, Fredrik Gustafsson, Fredrik Gunnarsson \\ Department of Electrical Engineering \\ Linköping University, SE-581 83 Linköping, Sweden \\ Email: [frida, fredrik, fred] disy.liu.se
}

\begin{abstract}
In many real-time applications, sample values and time stamps are delivered in pairs, where sampling times are non-uniform. Frequency analysis using non-uniform data occurs in various real life problems and embedded systems, such as vibrational analysis in cars and control of packet network queue lengths. Our contribution is to first overview different ways to approximate the Fourier transform, and secondly to give analytical expressions for how non-uniform sampling affects these approximations. The results are expressed in terms of frequency windows describing how a single frequency in the continuous time signal is smeared out in the frequency domain, or, more precisely, in the expected value of the Fourier transform approximation.
\end{abstract}

\section{INTRODUCTION}

Frequency analysis using non-uniform data occurs in many real life problems and in many embedded systems. For instance, in automotive applications, all signals in modern cars are taken from the CAN bus, where sensor observations and time stamps are delivered in pairs upon regular or event triggered requests. For chassi and tire vibration analysis, it is thus useful to gain full insight into how this affects frequency analysis. As an example, the wheel angular speed signals are event-based sampled in the time domain (uniformly sampled in the angular domain), and these signals are crucial for many control and informations systems in cars $[1,2]$. These signals are delivered with a time stamp, but the real sampling instants may differ with a random value causing a so called jitter sampling.

Another application of our focus is adaptive network queue control. The protocol in current Internet routers gives the queue length each time instant a packet arrives, but not when packets leave the queue. The router then sends an acknowledgment back to the sender that the packet is received. However, in active queue management (AQM), the router may decide not to send an acknowledgment if the queue is full or is likely to become full if the senders continue to send with the current rate. The basic idea in model-based AQM
[3] is to base the control principle on frequency analysis, or a model derived from frequency analysis. It has been empirically noted that the queue length in Internet routers contains frequency components, but the complicated interplay of the network makes an analytical approach intractable.

The idea is thus to consider the queue length $y(t)$ as a continuous time function, randomly sampled when packets of unequal sizes arrive. Classical approaches on queue theory derive distributions for the inter arrival times for packets of fixed sizes, ranging from simple Poisson processes to more recent self-similar processes [4], but these are not so suitable for control purposes.

Randomized sampling is described in $[5,6,7]$. Research is focused on how to choose sampling instants to maximize alias frequency suppression. In [8], an empirical motivation to add random jitter is given with some user guidelines. In [9], an algorithm for hardware implementation promising 40 times the bandwidth of the corresponding uniform sampling process. Algorithms for recovery of band limited signals are given in $[10,11]$. No illustration of the effects of leakage when nonuniform sampling is used have been found.

\section{OVERVIEW}

Consider a continuous time signal $y(t)$, which is non-uniformly sampled $y\left(t_{i}\right)$ at the time instants $t_{i}, i=1,2, \ldots N$, where we denote the sampling intervals $T_{i}=t_{i}-t_{i-1}$. Random additive sampling occurs when $T_{i}$ are independent random variables and jitter sampling when $t_{i}=i T+n_{i}$, where $n_{i}$ are random variables.

Our interest is focused on approximating the Fourier transform

$$
Y(f)=\int_{-\infty}^{\infty} y(t) e^{i 2 \pi f t} d t .
$$

We will first survey available methods that fall into one of two different approaches to approximate this continuous time integral:

1. A Riemann approximation approach, where the integrand $y(t) e^{i 2 \pi f t}$ is spline interpolated between the 
observations. For uniform sampling and a zero order spline (piece-wise constant integrand), the discrete Fourier transform (DFT) is obtained as a special case.

2. An interpolation approach, where the signal value is interpolated using splines or other basis functions. For a band-limited signal, the well-known sinc basis function expansion

$$
\begin{aligned}
y(t) & =\sum_{k=1}^{N} y(k T) \frac{\sin (\pi(t-k T) / T)}{\pi(t-k T) / T} \\
& =\sum_{k=1}^{N} y(k T) \operatorname{sinc}\left(\frac{t-k T}{T}\right)
\end{aligned}
$$

exists for uniform sampling. Note that this interpolation formula provides a system of linear equations, which is solvable for $y(k T), k=1,2, \ldots N$ for any set of non-uniform samples $y\left(t_{i}\right), i=1,2, \ldots, M$ whenever $M \geq N$, that is, when the average sampling rate is larger than or equal to the Nyquist rate.

These approaches will yield an approximation $\hat{Y}(f)$. Since this is a linear function of the observations $y\left(t_{i}\right)$, superposition applies. Thus, insight into the implications of nonuniform sampling is obtained by studying the approximation of a pure sinusoid $y(t)=\sin \left(2 \pi f_{0} t\right)$. The approximation can then be expressed as

$$
\begin{aligned}
\hat{Y}(f) & =W\left(\cdot ; t^{N}\right) * Y(f) \\
& =W\left(f-f_{0} ; t^{N}\right)-W\left(f+f_{0} ; t^{N}\right) .
\end{aligned}
$$

Here $t^{N}$ denotes the set of sampling points, and $*$ denotes convolution. For uniform sampling and a uniform (boxcar) time window, the frequency window becomes the periodic sinc-like function

$$
W\left(f ; t_{k}=k T\right)=e^{-i \pi f N T} \frac{\sin (\pi f N T)}{\sin (\pi f T)} .
$$

This function describes the leakage effects. Other data windows as Hamming, Hanning etc. gives other similar windows. Note that since these are all periodic functions, we have the well-known frequency ambiguity in uniform sampling. This is not the case for non-uniform sampling, where leakage and frequency ambiguity are intrinsic properties of the sampling instants, both revealed in the frequency window $W\left(f, f_{0} ; t^{N}\right)$.

The main contribution is then to characterize the expected value of this approximation for randomized sampling, where the exact sampling times $t_{k}$ are unknown, and only their distribution is known. The implication of randomized sampling is that the expected value of the Fourier transform approximation depends on the probability density function (pdf) $p\left(t^{N}\right)$ for the sampling instants, so we get

$$
\mathrm{E}(\hat{Y}(f))=W\left(f, f_{0} ; p\left(t^{N}\right)\right)-W\left(f,-f_{0} ; p\left(t^{N}\right)\right) .
$$

\section{FOURIER TRANSFORM APPROXIMATIONS}

The measurements, $y\left(t_{i}\right)$, are used to approximate the integrand, $I(t)$, or the original continuous signal, $y(t)$. These approximations are then used together with the definition of the continuous Fourier transform, (1), to produce an estimate, $\hat{Y}(f)$, of the transform for the measured signal. Three different transform approximations are presented: Extended Riemann approximation; Reconstruction of $y(t)$ using splines; and Reconstruction of bandlimited signals.

More elaborate calculations and an evaluation of the transform approximations can be found in [12].

Spline interpolation is done by connecting sample points, $f\left(t_{k}\right)$, with polynomials, $p_{k}^{n}(t)$, of order $n$. The continuous function approximation is defined as

$$
\hat{f}(t)=p_{k}^{n}(t), \quad t_{k-1}<t \leq t_{k} .
$$

The polynomial constants are defined by continuity demands at the sample points.

\subsection{Integrand interpolation}

Riemann approximation of an integral is approximation of the integrand with a piecewise constant function. Using the measurements, $y\left(t_{k}\right)$, the integrand $I(t)=y(t) e^{-i 2 \pi f t}$ can be approximated using higher order splines. Let $\hat{I}^{n}(t)$ be the continuous estimate of $I(t)$, based on measurements, $I\left(t_{k}\right)=y\left(t_{k}\right) e^{-i 2 \pi f t_{k}}$, and an $n$th order spline. The first two splines become

$$
\begin{aligned}
& \hat{I}^{0}(t)=I\left(t_{k}\right) \\
& \hat{I}^{1}(t)=\frac{I\left(t_{k}\right)-I\left(t_{k-1}\right)}{T_{k}}\left(t-t_{k}\right)+I\left(t_{k}\right),
\end{aligned}
$$

for $t_{k-1}<t \leq t_{k}$.

The Fourier transform estimate becomes

$$
\hat{Y}_{r a}^{n}(f)=\sum_{k=1}^{N} \int_{t_{k-1}}^{t_{k}} \hat{I}^{n}(t) d t .
$$

For the first orders of $n$, the explicit expressions become

$$
\begin{aligned}
\hat{Y}_{r a}^{0}(f) & =\sum_{k=1}^{N} I\left(t_{k}\right) T_{k}=\sum_{k=1}^{N} y\left(t_{k}\right) T_{k} e^{-i 2 \pi f t_{k}} \\
\hat{Y}_{r a}^{1}(f) & =\sum_{k=1}^{N} \frac{T_{k}}{2}\left(I\left(t_{k}\right)+I\left(t_{k-1}\right)\right) \\
& =\frac{1}{2} \sum_{k=1}^{N}\left(T_{k}+T_{k+1}\right) I\left(t_{k}\right) .
\end{aligned}
$$

The last equality demands that $T_{1} I\left(t_{0}\right)=0$ and $T_{N+1} I\left(t_{N}\right)=$ 0 , but the relation shows that the increased polynomial order merely changed the scaling of the integrand $I\left(t_{k}\right)$. The mean value of two subsequent inter sample times are used instead of only $T_{k}$. 


\subsection{Signal interpolation}

Spline interpolation of the signal can be done by connecting the sample points, $y\left(t_{k}\right)$ with polynomials, $p_{k}^{n}(t)$, of order $n$. The continuous function estimate is, as before, defined as

$$
\hat{y}^{n}(t)=p_{k}^{n}(t), \quad t_{k-1}<t<t_{k} .
$$

When $n=0, \hat{y}^{0}(t)$ is piece-wise constant. The explicit transform in this case becomes

$$
\begin{aligned}
\hat{Y}_{s p}^{0}(f) & =\sum_{k} y_{k} \int_{t_{k-1}}^{t_{k}} e^{-i 2 \pi f t} d t \\
& =\frac{i}{2 \pi f} \sum_{k=1}^{N} y_{k} e^{-i 2 \pi f t_{k}}\left(1-e^{i 2 \pi f T_{k}}\right) .
\end{aligned}
$$

Comparing $\hat{Y}_{s p}^{0}$ with Riemann approximation of the integral, $\hat{Y}_{r a}^{0}(9)$, shows that the difference lies in the scaling of the terms in the sum. Using a Taylor expansion for $e^{i 2 \pi f T_{k}}$ shows that $Y_{s p}^{0}$ scales the terms with

$$
\frac{i}{2 \pi f}\left(1-e^{-i 2 \pi f T_{k}}\right)=T_{k}+O\left(T_{k}^{2}\right),
$$

while $\hat{Y}_{r a}^{0}$ scales the terms with $T_{k}$. This means that

$$
\hat{Y}_{s p}^{0} \approx \hat{Y}_{r a}^{0}, \text { if } T_{k}<<1, \forall k
$$

For $n=1, \hat{y}^{1}(t)$ is piece-wise linear between the measurement points. The spline polynomials become

$$
p_{k}^{1}(t)=\frac{y_{k}-y_{k-1}}{T_{k}}\left(t-t_{k}\right)+y_{k}, k=1, \ldots, N .
$$

Letting $\alpha_{k}=\frac{y_{k}-y_{k-1}}{T_{k}}$ gives the transform approximation

$$
\begin{aligned}
\hat{Y}_{s p}^{1}(f) & =\sum_{k=1}^{N} \int_{t_{k-1}}^{t_{k}}\left[\alpha_{k}\left(t-t_{k}\right)+y_{k}\right] e^{-i 2 \pi f t} d t \\
& =\hat{Y}_{s p}^{0}(f) \\
& +\frac{1}{(2 \pi f)^{2}} \sum_{k=1}^{N} \alpha_{k} e^{-i 2 \pi f t_{k}}\left(1-e^{i 2 \pi f T_{k}}\right) \\
& +\frac{i}{2 \pi f} \sum_{k=1}^{N} \alpha_{k} T_{k} e^{-i 2 \pi f t_{k}} e^{i 2 \pi f T_{k}}
\end{aligned}
$$

The transform of $y_{k}$, for $n=1$, contains the transform of $y_{k}$ for $n=0$, the scaled transform of $\alpha_{k}$ for $n=0$ and a third term based on $\alpha_{k}$. The structure of the third term is very similar to the structure of a Riemann approximation. The novelty when introducing a higher order spline representation is shown in the last two terms.

For higher order splines the expressions become messier and no calculations have been performed for this case.

\subsection{Band-limited signals}

For bandlimited signals the sinc function can be used. Applying the assumption of an underlying sum of sinc's

$$
\hat{y}(t)=\sum_{k} c_{k} \operatorname{sinc}\left(a_{k}\left(t-b_{k}\right)\right)
$$

gives a straightforward calculation of the Fourier transform.

$$
\begin{aligned}
\hat{Y}_{\text {sinc }}(f) & =\sum_{k} c_{k} \int_{-\infty}^{\infty} \operatorname{sinc}\left(a_{k}\left(t-b_{k}\right)\right) e^{-i 2 \pi f t} d t \\
& =\sum_{k: f<\frac{a_{k}}{2}} \frac{1}{a_{k}} c_{k} e^{-i 2 \pi f b_{k}} .
\end{aligned}
$$

The signal is bandlimited to $\max _{k}\left(a_{k} / 2\right)$. After choosing $a_{k}$ and $b_{k}$, the amplitudes $c_{k}$ can be solved for from a linear equation system, $y\left(t_{i}\right)=\hat{y}\left(t_{i}\right), \forall i$. Placing the sinc's equidistantly is one option, i.e., $b_{k}=k T, a_{k}=\frac{1}{T}$. From (2), this case gives that $c_{k}=y(k T)$. This special transform approximation becomes

$$
\hat{Y}_{\text {sinc }}(f)= \begin{cases}T \sum_{k=1}^{N} c_{k} e^{-i 2 \pi f k T}, & f<\frac{1}{2 T}, \\ 0, & \text { otherwise. }\end{cases}
$$

\section{STOCHASTIC SAMPLING}

To find the window in (7) the expected value of $\hat{Y}_{s p}^{0}(f)$ will be calculated when

$$
y(t)=\sin \left(2 \pi f_{0} t\right)=\frac{1}{2 i}\left(e^{i 2 \pi f_{0} t}-e^{-i 2 \pi f_{0} t}\right)
$$

is sampled nonuniformly and the inter event times, $T_{k}$, have the probability density function $f_{T}(\tau)$. The details of the calculations have been left out and can be found in [12], as before. The samples, $y_{k}=y\left(t_{k}\right)=y\left(\sum_{n=1}^{k} T_{n}\right)$, become

$$
y_{k}=\frac{1}{2 i}\left(e^{i 2 \pi f_{0} \sum_{n=1}^{k} T_{n}}-e^{-i 2 \pi f_{0} \sum_{n=1}^{k} T_{n}}\right)
$$

Assuming that the inter sample times, $T_{k}$, are independent identically distributed stochastic variables, with probability density $f_{T}(\tau)$, the expected value of $\hat{Y}_{s p}^{0},(12)$, is calculated, using $y_{k}$ from (17), as

$$
\mathrm{E}\left[\hat{Y}_{s p}^{0}(f)\right]=\frac{1}{4 \pi f}\left(\chi\left(f, f_{0}\right)-\chi\left(f,-f_{0}\right)\right) .
$$

This means that the window (7) is

$$
W\left(f, f_{0}, p\left(t^{N}\right)\right)=\chi\left(f, f_{0}\right) /(2 \pi f) .
$$

The function $\chi\left(f, f_{0}\right)$ evaluates according to

$$
\begin{aligned}
& \chi\left(f, f_{0}\right)= \begin{cases}\left(\gamma\left(f, f_{0}\right)-\gamma\left(0, f_{0}\right)\right) \frac{1-\gamma\left(f, f_{0}\right)^{N}}{1-\gamma\left(f, f_{0}\right)} & \gamma\left(f, f_{0}\right) \neq 1 \\
\left(1-\gamma\left(0, f_{0}\right)\right) N & \gamma\left(f, f_{0}\right)=1\end{cases} \\
& \gamma\left(f, f_{0}\right)=\int_{\mathbf{R}} e^{-i 2 \pi\left(f-f_{0}\right) \tau} f_{T}(\tau) d \tau
\end{aligned}
$$



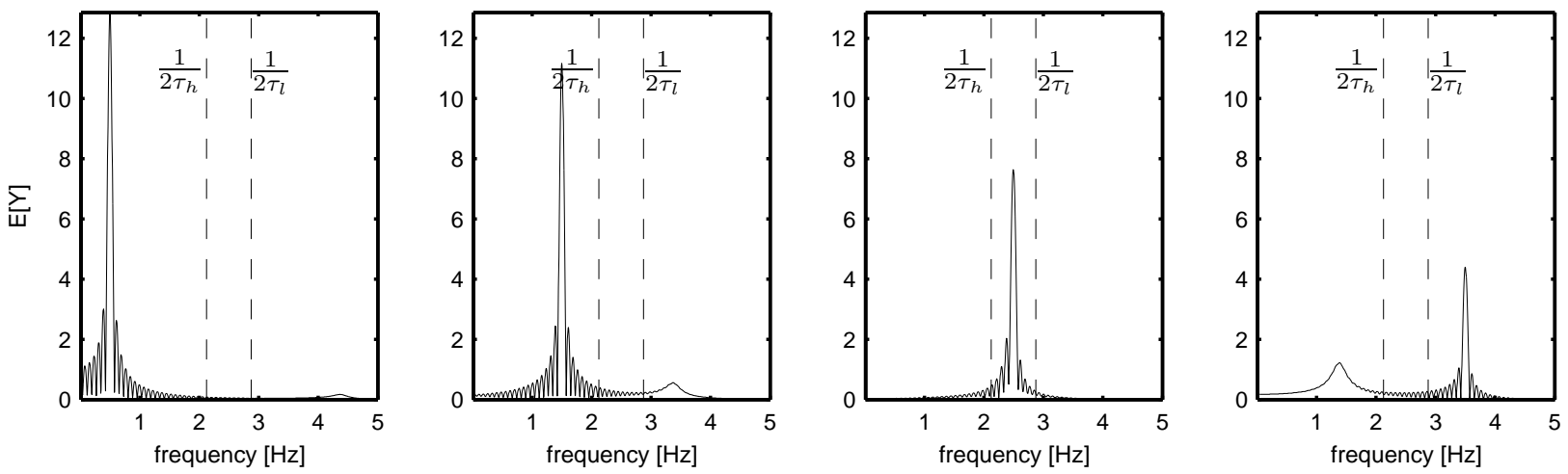

Fig. 1. $E\left[\hat{Y}_{s p}^{0}(f)\right]$ in (18), uniform distribution, $T_{k} \in\left[\tau_{l}, \tau_{h}\right]$, for the signal (16) with $f_{0}=[0.5,1.5,2.5,3.5]$.

The choice of sampling distribution affects the final transform through the expected values

$$
\begin{gathered}
\gamma\left(f, f_{0}\right)=\mathrm{E}_{T}\left[e^{-i 2 \pi\left(f-f_{0}\right) T}\right], \\
\gamma\left(0, f_{0}\right), \gamma\left(f,-f_{0}\right), \text { and } \gamma\left(0,-f_{0}\right),
\end{gathered}
$$

where $\mathrm{E}_{T}$ means expected value with respect to $T$. Figure 1 illustrates the window, (18), when $f_{T}$ describes a uniform distribution, $T_{k} \in\left[\tau_{l}, \tau_{h}\right]$. An exponential distribution with the same support gives a similar window.

Since $\hat{Y}_{s p}^{0} \approx \hat{Y}_{r a}^{0}$ for small inter event times and $\hat{Y}_{r a}^{0}=$ $\hat{Y}_{\text {sinc }}$ for uniform sampling and $f<\frac{1}{2 T}$, the analytical expression of $E\left[\hat{Y}_{s p}^{0}\right]$ is of great interest. It can be argued that the expected values for $\hat{y}_{r a}^{0}$ and $\hat{y}_{s i n c}$ will behave similar to $E\left[\hat{Y}_{s p}^{0}\right]$ for small $T_{k}$ 's and narrow distributions, respectively. It remains to investigate the extent of the correspondence.

\section{CONCLUSIONS}

This work presented an analytical expression for the expected value of a Fourier transform approximation based on additive random sampling. It was argued that the support of the inter sample times affects the alias suppression.

\section{REFERENCES}

[1] F. Gustafsson, S. Ahlqvist, U. Forssell, and N. Persson, "Sensor fusion for accurate computation of yaw rate and absolute velocity," in Society of Automotive Engineers World Congress, Detroit, 2001, number SAE 2001-01-1064.

[2] F. Gustafsson, M. Drevö, U. Forssell, M. Löfgren, N. Persson, and H. Quicklund, "Virtual sensors of tire pressure and road friction," in Society of Automotive Engineers World Congress, Detroit, 2001, number SAE 2001-01-0796.
[3] F. Gunnarsson, F. Gunnarsson, and F. Gustafsson, "Controlling internet queue dynamics using recursively identified models," in 42nd IEEE Conference on Decision and Control, Dec. 2003.

[4] S. Andersson, Hidden Markov Models - Traffic Modeling and Subspace Methods, Ph.D. thesis, Lund Institute of Technology, 2002.

[5] A. Papoulis, Signal Analysis, McGraw-Hill, 1977.

[6] I. Bilinskis and A. Mikelsons, Randomized Signal Processing, Prentice Hall, London, 1992.

[7] F. Marvasti, Zero-crossings and Nonuniform Sampling of Single and Multidimensional Signals and Systems, Nonuniform, 1987.

[8] D. M. Bland and A. Tarczynski, "Optimum nonuniform sampling sequence for alias frequency suppression," in IEEE International Symposium on Circuits and Systems, Jun 1997.

[9] F. Papenfuss, Y. Artyukh, E. Boole, and D. Timmermann, "Optimal sampling functions in nonuniform sampling driver designs to overcome the nyquist limit," in Acoustics, Speech, and Signal Processing, 2003., IEEE International Conference on, Apr 2003.

[10] F. Marvasti, "Nonuniform sampling theorem for bandpass signals at or below the nyquist density," IEEE Transactions on Signal Processing, Mar 1996.

[11] F. Marvasti, M. Analoui, and M. Gamshadzahi, "Recovery of signals from nonuniform samples using iterative methods," IEEE Transactions on Signal Processing, Apr 1991.

[12] F. Gunnarsson, "On modeling and control of network queue dynamics," Licenciate Thesis, No. 1048, Dep. of Electrical Engineering, Linköpings Universitet, 2003, URL: www.control.isy.liu.se/publications. 